\title{
Numerical Study of the Effect of Temperature on the Performance of a Silicon Heterojunction Solar Cell (HIT) in the Presence of Excitons
}

\author{
Ousmane Ngom ${ }^{1, ~ *}$, Modou Faye ${ }^{1}$, Mamadou Mbaye ${ }^{1}$, Cheikh Mbow ${ }^{2}$, Bassirou Ba ${ }^{1}$ \\ ${ }^{1}$ Department of Physics, Laboratory of Semiconductors and Solar Energy, Faculty of Science and Technology, University Cheikh Anta DIOP, \\ Dakar, Senegal \\ ${ }^{2}$ Department of Physics, Laboratory of Fluid Mechanics, Hydraulics and Transfers, Faculty of Science and Technology, University Cheikh \\ Anta DIOP, Dakar, Senegal
}

Email address:

ousmanengom460@gmail.com (O. Ngom), fayendiouma80@gmail.com (M. Faye)

${ }^{*}$ Corresponding author

\section{To cite this article:}

Ousmane Ngom, Modou Faye, Mamadou Mbaye, Cheikh Mbow, Bassirou Ba. Numerical Study of the Effect of Temperature on the Performance of a Silicon Heterojunction Solar Cell (HIT) in the Presence of Excitons. International Journal of Materials Science and Applications. Special Issue: Advanced Materials for Energy Storage and Conversion Applications. Vol. 8, No. 4, 2019 , pp. 56-67. doi: 10.11648/j.ijmsa.20190804.11

Received: July 10, 2019; Accepted: August 18, 2019; Published: September 6, 2019

\begin{abstract}
In this article, a detailed study of the physical phenomena in the base of a silicon heterojunction solar cell (HIT) is elaborated. To carry out this work we have established a mathematical model which is in the form of a system of two continuity equations. The latter are subjected to physical conditions of nature to define our field of study. This system of continuity equations is solved using a computational program in a digital programming language. Numerical analysis is used in this study because the mathematical system describing the transport phenomena of load carriers (electrons and excitons) in a silicon heterojunction photovoltaic cell is very complex. Thus, to facilitate numerical resolution, the dimensional parameters of the physical system are rendered dimensionless. The resulting dimensionless equations are discretized by the finite volume method. They are then implemented in a calculation program by an iterative line-by-line relaxation method of the Gauss-Siedel type. In addition, with a low density coupling coefficient $b=10^{-16} \mathrm{~cm}^{3} \mathrm{~s}^{-1}$ that depends on the material's properties, the influence of temperature on the diffusion lengths, on the carrier and photocurrent densities, and on the internal quantum yield is studied. This study is carried out using polychromatic illumination with ultraviolet, visible and infrared wavelengths.
\end{abstract}

Keywords: Heterojunction (HIT), Temperature, Excitons, Spectral Response, Quantum Performance

\section{Introduction}

The study of the effect of temperature on the solar cell has always aroused interest of some authors. Several researchers have conducted research in this area, including:

M. Faye, M. Niane, S. Ndiaye, O. Ngom, C. Mbow, B. Ba [1] have shown by considering a non-linear problem and voluminal coupling coefficient $b$ which depends on the temperature, the reduction of the effects of the high values wavelength on the total density of the photocurrent by those of the heat factor and the Fourier number.

M. Burgelman Minnaer et B. [2] which consider that the base is the sector that produces the majority of the photocurrent density.

Zh. Karazhanov [3], carried out his study in the space load area neglecting the electric field. He noticed that excitons have a diffusion length about 3 to 6 times smaller than electrons. Therefore, taking into account the excitons, the author found a significant increase in the current under dark and a decrease in the short-circuit current, the open-circuit voltage, the form factor and the efficiency.

As for M. Faye, C. Mbow, B. Ba [4-5], they also made a numerical study on the influence of the heating factor, the conversion rate and the density coupling coefficient of excitons on the photocurrent density in the base, including the space load area of a silicon homojunction cell. They used 
monochromatic light. They took into account the presence of a temperature-dependent electric field.

In the continuity of all this work, we studied the influence of temperature on the density of photocourant, on the internal quantum efficiency of a solar cell with silicon heterojunction (HIT) in the presence of excitons, under polychromatic illumination.

To do this, the following plan is drawn up:

First, we have developed a mathematical and numerical model with which we have highlighted a calculation program.

The latter leads to the results that will be commented on.

\section{Mathematical Modelling}

\subsection{Position of the Problem}

The study of a physical problem enabled us to establish a mathematical model, equations. These are governed by the phenomena of generation, recombination, diffusion and dissociation in a silicon heterojunction photovoltaic cell. We consider a silicon heterojunction cell of type $\left(n^{+} p\right)$, L length, illuminated by a mono (poly) chromatic light in the presence of excitons.

Our photocell consists of three parts

a) The transmitter: area highly doped in donor atoms (1, $\left.5.10^{19} \mathrm{~cm}^{-3}\right)$ b) Space Load Area (SLA): located between the transmitter and the base of an electric field. This allows the separation and acceleration of electron-hole pairs that arrive at the junction;

c) The base: almost neutral zone, doped in accepting atoms $\left(10^{17} \mathrm{~cm}^{-3}\right)$.

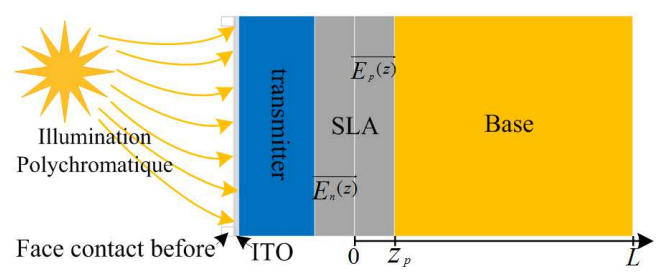

Figure 1. Structure of a silicon heterojonction solar cell (a-Si: H/c-Si) of type $\left(n^{+} p\right)$.

The mechanisms of generation-recombination, diffusion and dissociation govern the equations of continuity.

\subsubsection{Transport of Electrons in the Base}

In the doped type base (p), the minority carriers are charged electrons $(-q)$. These electrons come from free electron-hole pairs and excitons. The density of electrons obeys the law of storage of charges whose system of equations is given by:

$$
\left\{\begin{array}{l}
D_{e} \frac{\partial^{2} n_{e}}{\partial z^{2}}+\mu_{e} \frac{\partial\left[n_{e} E_{P}(z)\right]}{\partial z}=\frac{n_{e}-n_{e 0}}{\tau_{e}}+b N_{A}\left(n_{e}-n_{e 0}\right)-\frac{n_{x}-n_{x 0}}{\tau_{d}}-G_{e} \\
D_{x} \frac{\partial^{2} n_{x}}{\partial z^{2}}+\mu_{x} \frac{\partial\left[n_{x} E_{P}(z)\right]}{\partial z}=\frac{n_{x}-n_{x 0}}{\tau_{a}}-b N_{A}\left(n_{e}-n_{e 0}\right)+\frac{n_{x}-n_{x 0}}{\tau_{d}}-G_{x}
\end{array}\right.
$$

We describe the photo generation transport of the minority electrons in the base $\left(n_{e}\right)$ and electrons from the linked electron hole pairs: excitons $\left(n_{x}\right)$, by the system of differential equations (1) closed by initial conditions and boundaries.

\subsubsection{Boundary Conditions}

The evolution of a physical system depends on these boundary conditions. In the theoretical field of physics, we very often use mathematical models to solve physical phenomena. But mathematical equations applied to physics are often difficult or impossible to solve analytically or digitally without resorting to the physical conditions of nature often called boundary conditions.

a) For electrons from free electron-hole pairs:

$$
\left\{\begin{array}{l}
\left\lfloor D_{e} \frac{d n_{e}}{d z}\right\rfloor_{z=0}=S_{e}\left[n_{e}(0)-n_{e 0}\right] \\
\left\lfloor D_{e} \frac{d n_{e}}{d z}\right\rfloor_{z=L_{P}}=-S_{e}\left[n_{e}\left(L_{P}\right)-n_{e 0}\right]
\end{array}\right.
$$

b) For electrons from excitons:

$$
\left\{\begin{array}{l}
\left\lfloor D_{x} \frac{d n_{x}}{d z}\right]_{z=0}=S_{x}\left[n_{x}(0)-n_{x 0}\right] \\
\left\lfloor D_{x} \frac{d n_{x}}{d z}\right]_{z=L_{P}}=-S_{x}\left[n_{x}\left(L_{P}\right)-n_{x 0}\right]
\end{array}\right.
$$

Excitons being more present in organic semiconductors [6-8] where efficiency of organic photovoltaic cells rarely exceeds 5\% [9]. The highest occupied molecular orbital (energy) can be compared to the top of the valence band in inorganic semiconductors. The lowest unoccupied molecular orbital (energy) can be compared to the bottom of the conduction band in inorganic semiconductors.

In contrast, in inorganic semiconductors where we have bands with direct or indirect gap, the free electron-hole pairs are more numerous than excitons which are often neglected. Here the electron-hole pairs are weakly bound (excitons of Wanier Mott) [10] and can be easily separated.

Since silicon is an inorganic compound, therefore by hypothesis, the flux of photon generating electron-hole pairs linked can be expressed as a function of the flux of photon generating free electron-hole pairs by the following relationship: $\varphi_{x}(\lambda)=\frac{\varphi_{e}}{8}[2]$.

And $\varphi(\lambda)=\varphi_{e}(\lambda)+\varphi_{x}(\lambda)$, resulting in the following expressions: $\varphi_{e}(\lambda)=\frac{8}{9} \varphi(\lambda)$ and $\varphi_{x}(\lambda)=\frac{1}{9} \varphi(\lambda)$. With $\varphi(\lambda)$ being the photon flow that generates minority carriers in the base. 


\subsubsection{Diffusion Equations and Boundary Conditions Without Dimensions}

To facilitate the numerical resolution of these equations, it is often necessary to render them dimensionless in order to have homogeneous solutions.

By asking $z=z^{*} L, \quad w=w^{*} L, n_{e}=n_{e}^{*} N_{r}, n_{x}=n_{x}^{*} N_{r}$ and by replacing $z, w, n_{e}$ and $n_{x}$ by their values in the system of equations (1), we obtain the following dimensionless diffusion equations.

a) The electron transport equations and the adimensional boundary conditions of the base:

$$
\left\{\begin{array}{l}
F_{0 e} \frac{\partial^{2} n_{e}^{*}}{\partial z^{*^{2}}}+K_{e} \frac{\partial n_{e}^{*}\left(w_{p}^{*}-z^{*}\right)}{\partial z^{*}}=-A_{e}\left(n_{x}^{*}-n_{x 0}^{*}\right)+B_{e}\left(n_{e}^{*}-n_{e 0}^{*}\right)+\left(n_{e}^{*}-n_{e 0}^{*}\right)-G_{e}^{*} \\
F_{0 x} \frac{\partial^{2} n_{x}^{*}}{\partial z^{* 2}}+K_{x} \frac{\partial n_{x}^{*}\left(w_{p}^{*}-z^{*}\right)}{\partial z^{*}}=A_{x}\left(n_{x}^{*}-n_{e 0}^{*}\right)-B_{x}\left(n_{e}^{*}-n_{e 0}^{*}\right)+\left(n_{x}^{*}-n_{x 0}^{*}\right)-G_{x}^{*}
\end{array}\right.
$$

With:

$$
\begin{gathered}
F_{0 e}=\frac{D_{e} \tau_{e}}{L^{2}} ; K_{e}=\mu_{e} \tau_{e} \frac{E_{0}}{w} ; A_{e}=\frac{\tau_{e}}{\tau_{d}} ; B_{e}=b \tau_{e} N_{A} ; G_{e}^{*}=\frac{8}{9} \frac{\tau_{e}}{N_{r}} G \\
F_{0 x}=\frac{D_{x} \tau_{a}}{L^{2}} ; K_{x}=\mu_{x} \tau_{a} \frac{E_{0}}{w} ; A_{x}=\frac{\tau_{a}}{\tau_{d}} ; B_{x}=b \tau_{a} N_{A} ; G_{x}^{*}=\frac{1}{9} \frac{\tau_{a}}{N_{r}} G
\end{gathered}
$$

In the same way we obtain the initial conditions and the following dimensionless limits.

b) For electrons from free electron-hole pairs:

$$
\left\{\begin{array}{l}
\left\lfloor A_{d e} \frac{d n_{e}^{*}}{d z^{*}}\right]_{z^{*}=0}=\left[n_{e}^{*}(0)-n_{e 0}^{*}\right] \\
\left.\qquad \begin{array}{l}
d n_{e}^{*} \\
A_{L e}^{*}
\end{array}\right]_{z^{*}=L_{p}^{*}}=-\left[n_{e}^{*}\left(L_{P}\right)-n_{e 0}^{*}\right]
\end{array}\right.
$$

c) For electrons from excitons:

$$
\left\{\begin{array}{l}
\left\lfloor A_{d x} \frac{d n_{x}^{*}}{d z^{*}}\right\rfloor_{z^{*}=0}=\left[n_{x}^{*}(0)-n_{x 0}^{*}\right] \\
\qquad\left.A_{L x} \frac{d n_{x}^{*}}{d z^{*}}\right|_{z^{*}=L_{p}^{*}}=-\left[n_{x}^{*}\left(L_{p}^{*}\right)-n_{x 0}^{*}\right]
\end{array}\right.
$$

With: $A_{d e}=A_{L e}=\frac{D_{e}}{S_{e} L} ; A_{d x}=A_{L x}=\frac{D_{x}}{S_{x} L}$.

Some parameters such as electron scatter coefficients and excitons of these equations depend on temperature. Since the equations are non-linear, the result is that the calculation must be self-consistent and not present an analytical solution. Therefore, a numerical resolution of these equations is required.

\subsection{Digital Modelling}

To obtain a numerical solution to our problem, we must transform the differential equations of the mathematical model into a system of algebraic equations obtained after discretization. We have chosen the law of power as the method of discretization. This method involves integrating equations into a discrete set called control volume.

Then we will rewrite the system in the following conservative form:

$$
\begin{aligned}
& \frac{\partial}{\partial z} \psi_{k}\left(n_{k}\right)=\mathfrak{I}\left(z, n_{k}, n_{l}\right) \\
& \psi_{k}\left(n_{k}\right)=v_{k} n_{k}-F_{0 k} \frac{\partial n_{k}}{\partial z}
\end{aligned}
$$

The indices $\mathrm{k}$ and $\mathrm{l}$ are relative to electrons and excitons and are such that:

If $k=e$ then $l=x$ and if $k=x$ then $l=e$.

Table 1 below represents the different values of variables $v_{k}, F_{0 k}$ and $\mathfrak{I}\left(z, n_{k}, n_{l}\right)$ for each diffusion equation.

To lighten the expressions in the table, we decided to rewrite the dimensionless quantities without stars.

Table 1. Expressions of quantities in the conservative equation (4).

\begin{tabular}{lll}
\hline Base & & \\
\hline Coefficients & For electrons & For excitons \\
\hline$v_{k}$ & $-K_{e}(w-z)$ & $-K_{x}(w-z)$ \\
$F_{0 k}$ & $F_{0 e}$ & $F_{0 x}$ \\
$\mathfrak{I}\left(z, n_{k}, n_{l}\right)$ & $A_{e}\left(n_{x}-n_{x 0}\right)-B_{e}\left(n_{e}-n_{e 0}\right)-\left(n_{e}-n_{e 0}\right)+G_{e}$ & $-A_{e}\left(n_{x}-n_{x 0}\right)+B_{e}\left(n_{e}-n_{e 0}\right)-\left(n_{x}-n_{x 0}\right)+G_{x}$ \\
\hline
\end{tabular}




\subsubsection{Meshing and Discretization of the Domain}

The adimensionalization allowed us to transform our field of study between $z=0$ and $z=L_{p}$ into a continuous segment of dimension 1 that we will approach in a series of $I_{m}$ points (nodes) of abscissa rated $z_{i}$.

Distributions of electrons and excitons are very sensitive to the surface phenomenon, a fine mesh in the vicinity of $z=0$, $z=z_{p}$ and $z=L_{p}$ is adopted.

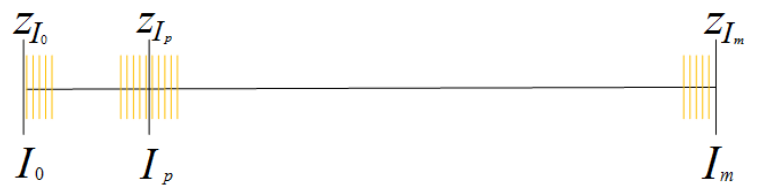

Figure 2. Area of study meshing

We will therefore use a trigonometric type mesh in the different fields: the space loading zone consists essentially of crystalline silicon of thickness $z=w_{p}$ and the base consists of crystalline silicon of thickness $z=L_{p}-w_{p}$. Positions are marked by depth $z_{i}$.

In these relationships, index $i=1$ denotes the interface between a-Si: $\mathrm{H}(\mathrm{n})$ and $\mathrm{c}-\mathrm{Si}(\mathrm{p})(z=0), I_{p}$ is the index that locates the position of the interface between the space load zone and the base $\left(z=w_{p}\right)$ and $I_{m}$ the position of the rear face where $\left(z=L_{p}\right)$.

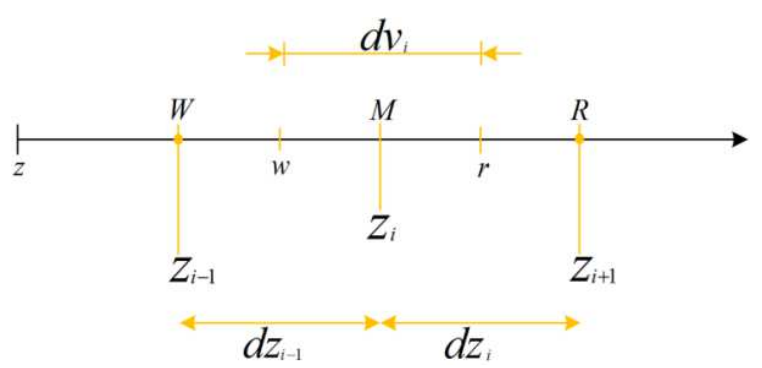

Figure 3. Discretization by the finite volume method.

The discretization steps are then given by:

$$
d z_{i}=z_{i+1}-z_{i}
$$

The segment centred around the $z_{i}$ node called the "control volume" and delimited by the nodes (w) and (r) is defined by $d v_{i}$ :

$$
\begin{aligned}
& d v_{i}=\frac{1}{2}\left(z_{i}-z_{i-1}\right) \\
& \left(v_{k}\right)_{r} n_{k, r}-\left(F_{0 k}\right)_{r} \frac{n_{k, R}-n_{k, M}}{(\delta z)_{r}}-\left(v_{k}\right)_{w} n_{k, w}+\left(F_{0 k}\right)_{w} \frac{n_{k, M}-n_{k, W}}{(\delta z)_{w}}=d v_{i} \cdot \overline{\mathfrak{I}\left(z, n_{k}, n_{l}\right)}
\end{aligned}
$$

If we consider $z_{i}$ abscissa knot, we note:

$$
(\delta z)_{r}=z_{i+1}-z_{i}
$$

and

$$
(\delta z)_{w}=z_{i}-z_{i-1}
$$

\subsubsection{Discretizations of the Equations}

To discreet the equations of the mathematical model that describes our physical system, we will use the finite volume method.

The finite volume method is based on a control volume approach and the formulation of an equation that often includes the following terms:

a) Unsteady term

b) Convective term

c) Diffusif term

d) Source term

In the case of this section, the unstable term will not be considered. In addition, some time-dependent optoelectric phenomena will be studied with time parameters fixed to appropriate values that correspond to our field of study (around $\mu s$ and $n s$ ). Let us now integrate the system into the control volume $d v_{i}$ it comes:

$$
\left[\psi_{k}\left(n_{k}\right)\right]_{r}-\left[\psi_{k}\left(n_{k}\right)\right]_{w}=d v_{i} \cdot \overline{\mathfrak{I}\left(z, n_{k}, n_{l}\right)}
$$

With $\overline{\mathfrak{I}\left(z, n_{k}, n_{l}\right)}$ the mean value of $\mathfrak{I}\left(z, n_{k}, n_{l}\right)$ defined by:

$$
\overline{\mathfrak{I}\left(z, n_{k}, n_{l}\right)}=\frac{1}{d v_{i}} \int_{w}^{r} \mathfrak{I}\left(z, n_{k}, n_{l}\right) d z
$$

To evaluate the gradients of the $n_{k}$ densities delimiting the control volume, we choose an interpolation between neighbouring nodes.

$$
\begin{aligned}
& \left(F_{0 k} \frac{\partial n_{k}}{\partial z}\right)_{r}=\left(F_{0 k}\right)_{r} \frac{n_{k, R}-n_{k, M}}{(\delta z)_{r}} \\
& \left(F_{0 k} \frac{\partial n_{k}}{\partial z}\right)_{w}=\left(F_{0 k}\right)_{w} \frac{n_{k, M}-n_{k, W}}{(\delta z)_{w}}
\end{aligned}
$$

We can rewrite Equation (11) by taking into account the expressions (13) and (14). Then we get:

There are different schematics to approximate the values of the $n_{k}$ functions: centred differences, upwind, hybrid, power 
law, 2nd order upwind, QUICK etc.

In our work, we chose the law of power because of its stability and precision.
By posing the expressions of new coefficients represented in the following Table 2.

Table 2. Expressions of quantities in discrete equations.

\begin{tabular}{llllll}
\hline Coefficients & $\boldsymbol{D}_{\boldsymbol{w}, \boldsymbol{k}}$ & $\boldsymbol{D}_{\boldsymbol{r}, \boldsymbol{k}}$ & $\boldsymbol{F}_{\boldsymbol{w}, \boldsymbol{k}}$ & $\boldsymbol{F}_{\boldsymbol{r}, \boldsymbol{k}}$ & $\boldsymbol{P}_{\boldsymbol{w}, \boldsymbol{k}}$ \\
\hline Expressions & $\frac{\left(F_{0 k}\right)_{w}}{(\delta z)_{w}}$ & $\frac{\left(F_{0 k}\right)_{r}}{(\delta z)_{r}}$ & $\left(V_{k}\right)_{w}$ & $\left(V_{k}\right)_{r}$ & $\frac{\left(V_{k}\right)_{w} \cdot(\delta z)_{w}}{\left(F_{0 k}\right)_{w}}$
\end{tabular}

Finally, a discrete equation is obtained for the variable $n_{k}$ of the carrier density in the general form of equation (16) :

$$
-a_{w, k} \cdot n_{k, i-1}+a_{M, k} \cdot n_{k, i}-a_{r, k} \cdot n_{k, i+1}=S_{m, k}
$$

The source term is uniform and linear: $S=S_{m, k}-S_{M, k} n_{k, i}$ in the control volume. We can therefore express it by equation (17) :

$$
d v_{i} \cdot \overline{\mathfrak{I}\left(z_{i}, n_{k}, n_{l}\right)}=d v_{i} \cdot\left(\overline{\mathfrak{I}_{m, k}}-\overline{\mathfrak{I}_{M, k}} \cdot n_{k, i}\right)=S_{m, k}-S_{M, k} \cdot n_{k, i}
$$

Table 3. Expressions of the quantities appearing in the source terms of the equations of continuities of electrons and excitons.

\begin{tabular}{lll}
\hline & $\overline{\mathfrak{I}_{\boldsymbol{M}, \boldsymbol{k}}\left(\boldsymbol{z}_{\boldsymbol{i}}, \boldsymbol{n}_{\boldsymbol{k}}, \boldsymbol{n}_{\boldsymbol{l}}\right)}$ & $\overline{\mathfrak{I}_{\boldsymbol{m}, \boldsymbol{k}}\left(\boldsymbol{z}_{\boldsymbol{i}}, \boldsymbol{n}_{\boldsymbol{k}}, \boldsymbol{n}_{\boldsymbol{l}}\right)}$ \\
\hline$k=e$ et $l=x$ & $1+B_{e}$ & $n_{e 0}\left(1+B_{e}\right)+A_{e}\left(n_{x}-n_{x 0}\right)+G_{e}$ \\
$k=x$ et $l=e$ & $1+A_{x}$ & $n_{x 0}\left(1+A_{x}\right)+B_{x}\left(n_{e}-n_{e 0}\right)+G_{x}$ \\
\hline
\end{tabular}

\subsubsection{Discretization of Initial and Boundary Conditions}

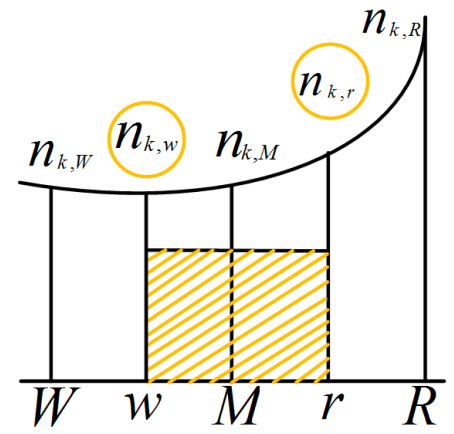

Figure 4. Power law diagram mesh.

In the same way as in the previous resolution of the diffusion equations of electrons and excitons, we will rewrite the conditions at the initial and final limits respectively in the form of two conservative equations; then he comes:

$$
\left\{\begin{array}{l}
\left\lfloor A_{d k} \frac{d n_{k}}{d z}\right\rfloor_{z=0}=\left[n_{k}(0)-n_{k 0}\right] \\
\left\lfloor A_{L k} \frac{d n_{k}}{d z}\right\rfloor_{z=L_{p}}=-\left[n_{k}\left(L_{P}\right)-n_{k 0}\right]
\end{array}\right.
$$

To solve the first and last terms of these equations, we need a Taylor serial development of the $n_{k}$ derivative as a function of $z$ in the first order.

Let us consider the point of abscissa $z_{i}$ as the point of origin of discrete space then:

$$
n_{k}\left(z_{i+1}\right)=n_{k}\left(z_{i}\right)+\delta z \frac{\partial n_{k}\left(z_{i}\right)}{\partial z}+0\left(\delta z^{2}\right)
$$

$$
n_{k}\left(z_{i-1}\right)=n_{k}\left(z_{i}\right)-\delta z \frac{\partial n_{k}\left(z_{i}\right)}{\partial z}+0\left(\delta z^{2}\right)
$$

We deduce from this the approximate expressions of the partial derivatives of $n_{k}$ as a function of the thickness

$$
\begin{gathered}
\frac{\partial n_{k}\left(z_{i}\right)}{\partial z}=\frac{1}{\delta z}\left(n_{k, i+1}-n_{k, i}\right) \\
\frac{\partial n_{k}\left(z_{i}\right)}{\partial z}=\frac{1}{\delta z}\left(n_{k, i}-n_{k, i-1}\right)
\end{gathered}
$$

By making the difference between relations (21) and (22), we have equation (23) which is obtained by a centered diagram of order 2 .

$$
\frac{\partial n_{k}\left(z_{i}\right)}{\partial z}=\frac{1}{2 \delta z}\left(n_{k, i+1}-n_{k, i-1}\right)
$$

This expression, applied to our boundary conditions, allows us to create a fictitious node in $i=I_{0}$. By taking into account relationships (21), (22) and (23), we get the boundary conditions represented in the equation system (24).

Finally, we will establish the complete matrix system that approaches our problem.

\subsubsection{Numerical (Algebraic) Model of Continuity Equations in the Base}

$\begin{cases}b_{1, k} n_{k, I_{0}}-C_{1, k} n_{k, I_{0}-1}=d_{1, k} & i=I_{0} \\ -a_{w, k} n_{k, i-1}+a_{M, k} n_{k, i}-a_{r, k} n_{k, i-1}=S_{m, k} & I_{0} \leq i \leq I_{m}-1 \\ -n_{k, I_{m}-1}+b_{m, k} n_{k, I_{m}}=d_{m, k} & i=I_{m}\end{cases}$

With here; 


$$
\begin{gathered}
b_{1, k}=a_{M 1, k}-2 \cdot C_{d k} \cdot a_{w 1, k} ; \quad c_{1, k}=a_{r 1, k}-a_{w 1, k} ; \\
d_{1, k}=S_{1, k}-2 \cdot C_{d k} \cdot a_{w 1, k} \cdot n_{k 0} \\
a_{m, k}=1 ; b_{m, k}=1+C_{L k} ; \quad d_{m, k}=C_{L k} \cdot n_{k 0}
\end{gathered}
$$

The $a_{w, k}, a_{M, k}$ and $a_{r, k}$ coefficients are evaluated at the control volume interfaces using the power law schema. They represent the combined conduction-diffusion flows. Expressions of these coefficients are given in the studies [11, $12]$.

\subsection{The Photocurrent Density of the Carriers}

The photocurrent density consists of the diffusion current due to the diffusion of the carriers via the photons and the conduction current due to the effect of the electric field of the space load zone.

\subsubsection{Current of Conduction}

In a material, the charged electrons collide with the atoms of the crystalline network. Each shock corresponds to a loss of energy. Between two shocks, the electrons are accelerated uniformly in the opposite direction of the electric field $E(z)$ of the SLA with a speed $\vartheta(z)= \pm \mu . E(z)$. The photocurrent density is then written:

a) For electrons:

$$
J_{c, e}=q \cdot n_{e} \cdot \mu_{e} \cdot E(z)
$$

b) For excitons:

$$
J_{c, x}=q \cdot n_{x} \cdot \mu_{x} \cdot E(z)
$$

The speeds $\vartheta_{e}(z)$ and $\vartheta_{x}(z)$ are proportional to the electric field. The sign of the mobility coefficients of holders $\mu_{e}(z)$ et $\mu_{x}(z)$ depends on their loads $(-q)$.

\subsubsection{Diffusion Current}

Fick's law, which is an experimental law established in 1856 by Adolphe Fick, states that: the number of particles per unit of time and volume $(\phi)$, is defined by the equation: $\phi=-D \frac{\partial n}{\partial z}$.

From this expression we can deduce the photocurrent densities of electrons and excitons.

a) For electrons:

$$
J_{d, e}=q \cdot D_{e} \cdot \frac{d n_{e}}{d z}
$$

b) For excitons:

$$
J_{d, x}=q \cdot D_{x} \cdot \frac{d n_{x}}{d z}
$$

The photocurrent densities of electrons and excitons are the sum of the two contributions: the diffusion currents and the conduction currents. c) For electrons:

$$
J_{e}=q \cdot n_{e} \cdot \mu_{e} \cdot E(z)+q \cdot D_{e} \cdot \frac{d n_{e}}{d z}
$$

d) For excitons:

$$
J_{x}=q \cdot n_{x} \cdot \mu_{x} \cdot E(z)+q \cdot D_{x} \cdot \frac{d n_{x}}{d z}
$$

The result of the photocurrent densities of electrons and excitons is the total photocurrent density of the carriers in the base:

$$
J=J_{e}+J_{x}
$$

\subsection{Spectral Response and Quantum Performance}

The spectral response is similarly the ratio of the intensity $I(\lambda)$ generated by the cell to the incident power $P_{i}(\lambda)$, for each wavelength. It is therefore a question of illuminating the cell and measuring the current it delivers

$$
S R=\frac{I(\lambda)}{P_{i}(\lambda)}=\frac{I(\lambda) / S}{\varphi_{i}(\lambda)}=\frac{J(\lambda)}{\varphi_{i}(\lambda)}
$$

Where $\varphi_{i}(\lambda)=\varphi_{0}(\lambda)$ is the incident photon flow and $\mathrm{S}$ is the cell surface. To calculate the flux of absorbed photon $\varphi_{a}(\lambda)$, the difference is made between the flux of photon at the input of the material (the illuminated face) and the flux of photon transmitted to a depth in the base.

It is generally this quantity that is measured and which makes it possible to calculate the quantum yield.

$$
\operatorname{EQE}(\lambda)=\frac{J(\lambda)}{q \cdot \lambda \cdot \varphi_{0}(\lambda)}
$$

One distinguishes external quantum yield, from internal quantum yield in which the flux of photon absorbed by the active layer of the cell is taken into account.

The expression presented here takes into account optical losses such as reflection or transmission through the cell: this is the external quantum yield.

Optical losses can be corrected to give the internal quantum yield that takes into account the characteristics specific to the cell (diffusion, surface and volume recombinations and/or absorption of the material under consideration).

By definition, $\mathrm{A}$ is the ratio of the number of photon photons generated to the number of photon absorbed. It can also be defined as the ratio between the external quantum yield and the absorption coefficient of the thickness layer $\mathrm{z}$.

$$
I Q E=\frac{E Q E(\lambda)}{A(\lambda)}
$$

With here: $A(\lambda)$ being the absorption coefficient of the thickness layer $z$. 


\section{Results and Discussions: The Influence of Temperature on the Optoelectric Parameters of Doped Crystalline Silicon, Type (p)}

Temperature is a very important parameter and its behaviour cannot be neglected in solar cells. The performance of a silicon solar cell is highly temperature sensitive. The latter acts on the molecular structure (the crystalline network) of silicon by exciting electrons and reducing the energy of the absorption gap.

In this article, we set out to study the effect of temperature in the base of a silicon heterojunction solar cell (HIT), often referred to as a high-efficiency solar cell. The basis of the HIT cell is the c-Si (p) with a thickness $L=0,025 \mathrm{~cm}$ and a

$$
k_{b}=1,380662 \cdot 10^{-23} J \cdot K^{-1} ; c=2,997924562.10^{8} m \cdot s^{-1} ; h=6,626176.10^{-34} J \cdot s^{-1} ; \varepsilon_{0}=8,842 \cdot 10^{-12}
$$

\subsection{Length of Diffusion of Load Bearers}

Figures 1 and 2 shows the diffusion lengths of electrons and excitons as a function of temperature.

The maximum diffusion length of electrons is about six times greater than that of excitons (Figure 6). At temperatures below $173 \mathrm{~K}$, excitons diffuse more than electrons. While at temperatures ranging from $173 \mathrm{~K}$ to $500 \mathrm{~K}$, electrons are more diffusible than excitons (Figure 5). In addition, we noted in Figure $5 \mathrm{~b}$ that the temperature has an antagonistic effect on the diffusion lengths of the two types of load carriers: electrons and excitons. That is, the increase of one leads to a decrease of the other and vice versa.

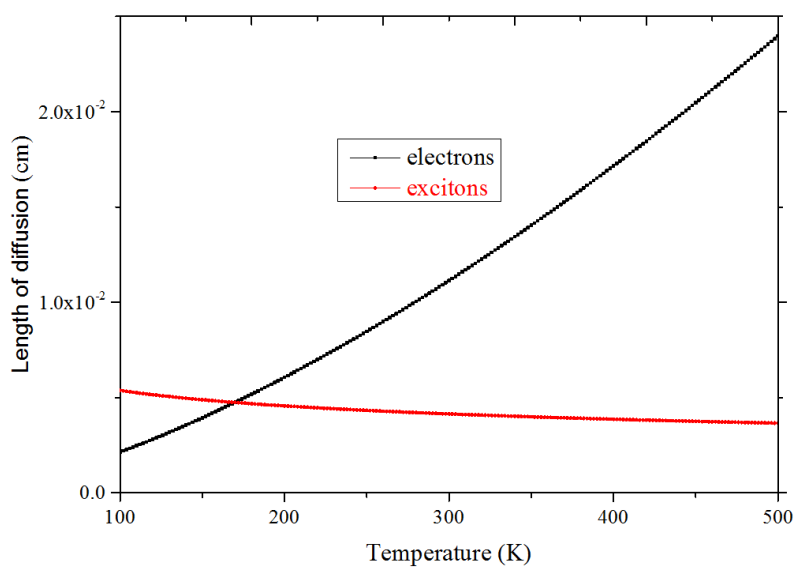

Figure 5. Electron and exciton diffusion lengths as a function of temperature.

Since excitons are in an excited state and are weakly related Wannier-Mott excitons. As a result, the lower the temperature, the more stable the excitons are and can easily spread. On the other hand, the higher the temperature, the more unstable the molecules in the cell, especially excitons that are already on excited levels.

In addition, at low temperatures, only those electrons that have enough energy to be allowed to rise above the forbidden band are scattered. But as the temperature increases, the electrons propagate deep in the cell, because in addition to the doping rate $N_{A}=1,14 \cdot 10^{17} \mathrm{~cm}^{-3}$. The emitter consists of a-Si: $\mathrm{H}$ (n) very strongly doped into donor atoms: $N_{D}=1,5.10^{19} \mathrm{~cm}^{-3}$, recombinant velocities of electrons and excitons: $S_{e}=S_{x}=6.10^{6} \mathrm{~cm} \cdot \mathrm{s}^{-1}$. Taking into account the actual mass of electrons $m_{e}=2,85689.10^{-31} \mathrm{Kg}$, we were able to assess the intrinsic parameters on which the mass depends. Indeed, excitons in inorganic semiconductors being pseudo particles whose electron-hole pairs are weakly bound; therefore, we can choose a very low-density coupling coefficient $\left(b_{v}=10^{-16} \mathrm{~cm}^{3} \cdot \mathrm{s}^{-1}\right)$ in the c-Si (p). In addition to the physical parameters related to the study and the intrinsic parameters, we can give the values of the physical constants involved in this work:

energy of sufficiently charged electrons, the temperature causes a reduction of the gap energy thus favoring the movement of the carriers free charge (electrons).

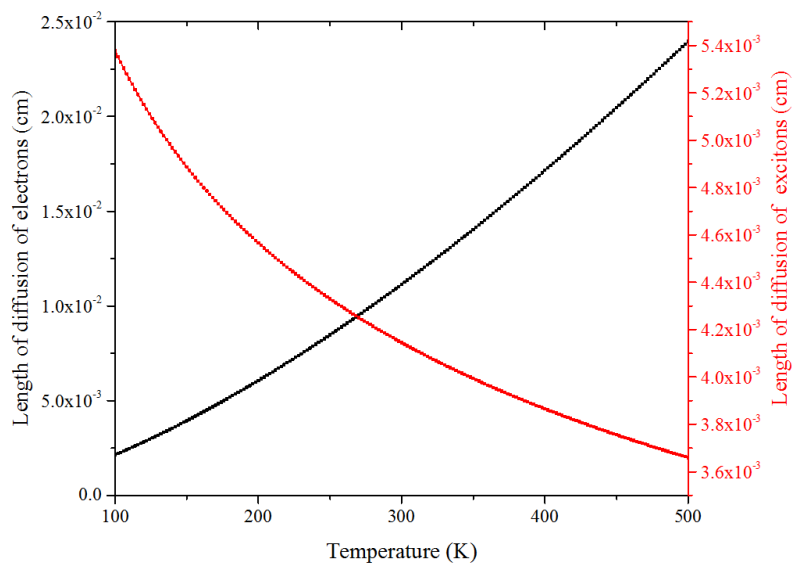

Figure 6. Electron and exciton diffusion lengths as a function of temperature.

The evolution of the diffusion lengths of electrons and excitons as a function of temperature allowed us to understand the movements of the carriers in depth in the cell, but does not edify us on the real effect of temperature on the photovoltaic cell. To better understand the influence of temperature, we will evaluate it on the density of the load carriers.

\subsection{The Density of the Carriers of Charge}

Figures 7 and 8 respectively represent the density of electrons and the density of excitons as a function of the depth of crystalline silicon for different temperature values.

The densities are evaluated according to the thickness in the base and in part of the space load zone made up of the P-type doped crystalline silicon. We see an increase in the density of the carriers (electrons and excitons) up to a thickness value in the base equal to $0.025 \mathrm{~cm}$ and a gradual decrease.

Nevertheless, for different values of the temperature, we noticed that an increase of the temperature causes a decrease of the density of the electrons (Figure 7) and an increase of the 
density of the excitons (Figure 8).

As with diffusion length, variations in electron and exciton densities are closely related. Since the load carriers are subjected to the action of the electric field in the space charge zone, the electron-hole pairs are separated and propelled into the base (generation). In fact, in the presence of the electric field, the density of the carriers increases to a certain depth in the base at densities of electrons and excitons that reach respectively maximum values of $7.10^{18} \mathrm{~cm}^{-3}$ and $10^{18} \mathrm{~cm}^{-3}$ at a thickness of $0,0125 \mathrm{~cm}$. Then, some carriers are subjected to phenomena of recombination and dissociation (excitons cases), so we obtain minimum values of densities at the level of the back side of the base.

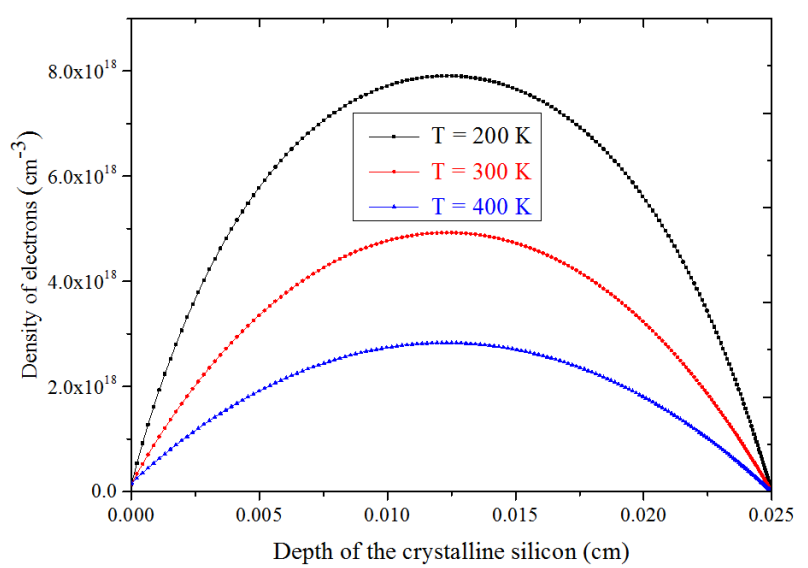

Figure 7. The density of electrons as a function of the depth of crystalline silicon with different temperature values.

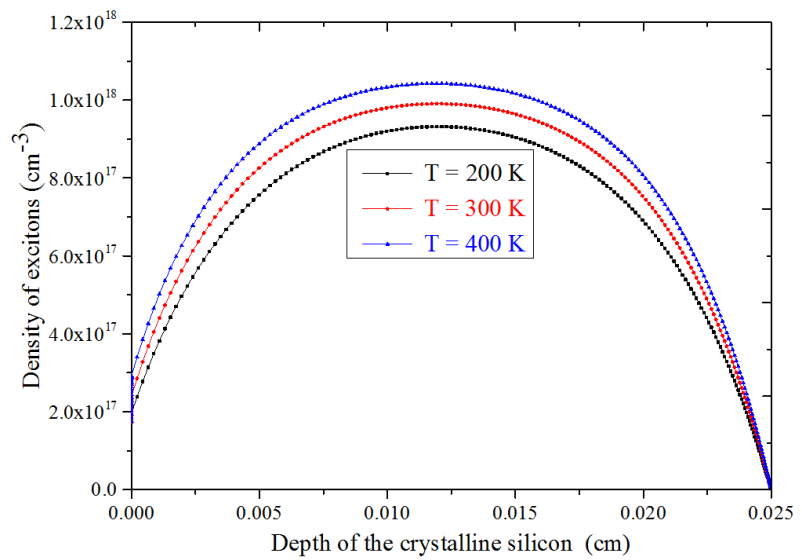

Figure 8. The density of excitons as a function of the depth of crystalline silicon with different temperature values.

In addition, at low temperatures $(200 \mathrm{~K})$, molecules in the cell are less subject to external influences such as thermal excitation. As the temperature rises, the molecules in the cell become more and more agitated. This agitation of thermal origin causes some electrons to be in excited positions. As a result, the density of excitons increases to the detriment of that of electrons.

In summary, we were able to assess the amount of carriers in a part of the cell made up of c-Si (P). But it is not enough to have a very large number of carriers in the cell to predict its ability to produce energy because in addition to the diffusion and the number of carriers in the cell, the movement of free and bound electrons must be ordered, hence the importance of studying the photocurrent density of electrons and excitons.

\subsection{Photocurrent Density of Charge Carriers}

Figures 9-20 provide information on the photocurrent density of electrons, that of excitons and on the total density as a function of the depth of crystalline silicon for different temperature values.

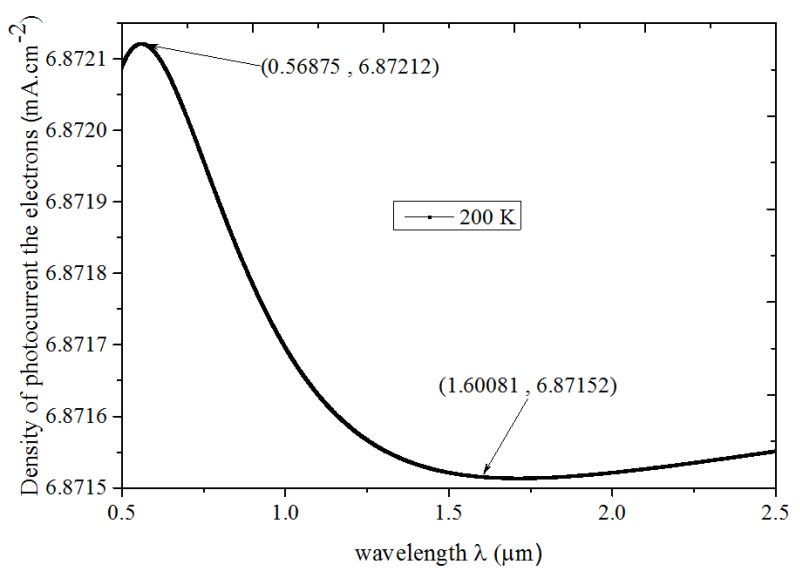

Figure 9. Electron photocurrent density as a function of wavelength $(\lambda)$ at $T=200 \mathrm{~K}$

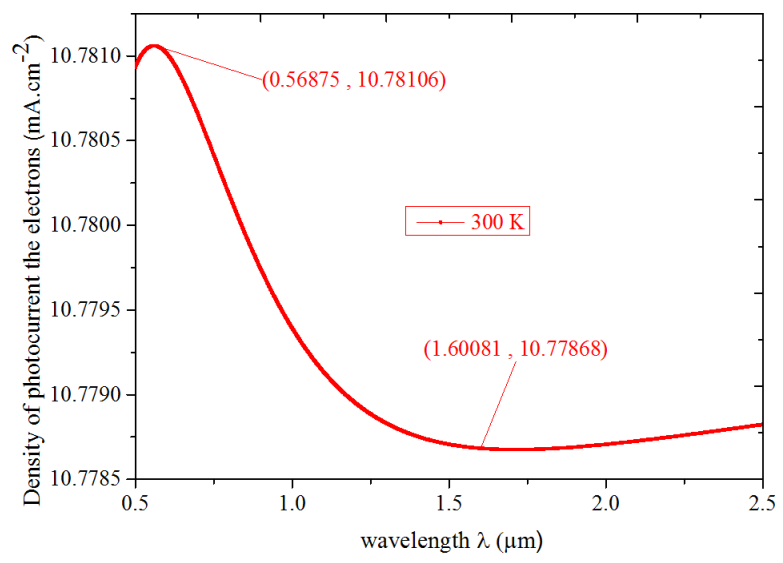

Figure 10. Electron photocurrent density as a function of wavelength $(\lambda)$ at $T=300 \mathrm{~K}$.

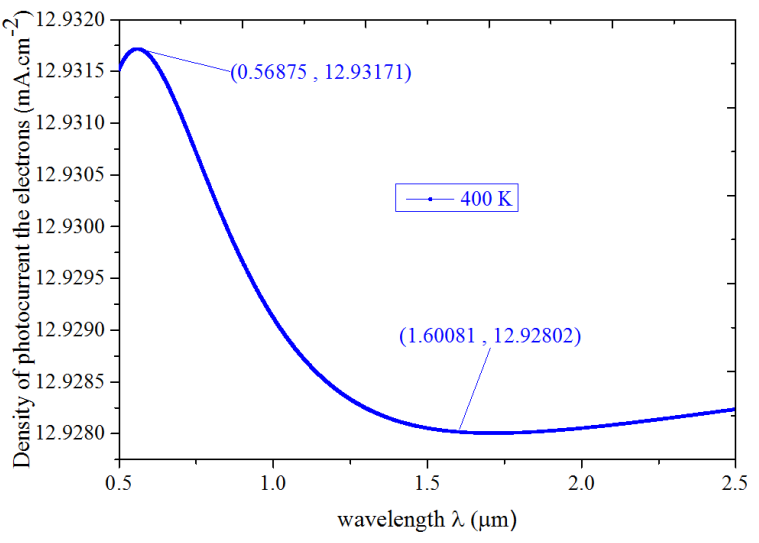

Figure 11. Electron photocurrent density as a function of wavelength $(\lambda)$ at $T=400 \mathrm{~K}$. 
In this part, the cell is illuminated by a polychromatic light with wavelengths between $0,5 \mu \mathrm{m}$ and $2,5 \mu \mathrm{m}$. When we evaluate the photocurrent density as a function of the wavelength, we observe a gradual decrease in the photocurrent density to a value of $\lambda=1,6 \mu \mathrm{m}$. Beyond this value, the photocurrent density of the electrons, that of the excitons and the total density remain practically constant.

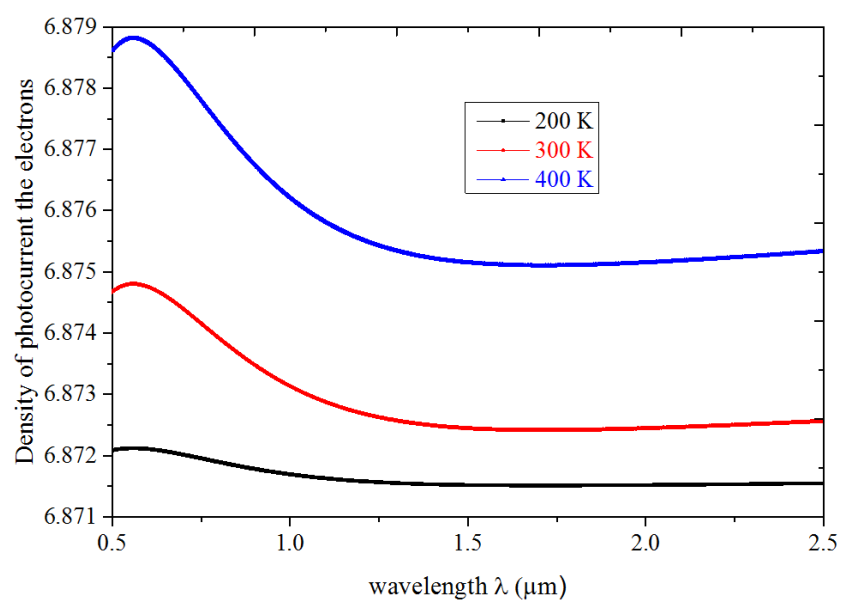

Figure 12. Electron photocurrent density as a function of wavelength $(\lambda)$ for different temperature values.

We have also noticed that crystalline silicon only responds to wavelengths between $0,6 \mu \mathrm{m}$ and $1,6 \mu \mathrm{m}$. Outside of this wavelength range, the photocurrent density is virtually unchanged (see Figures 9-20).

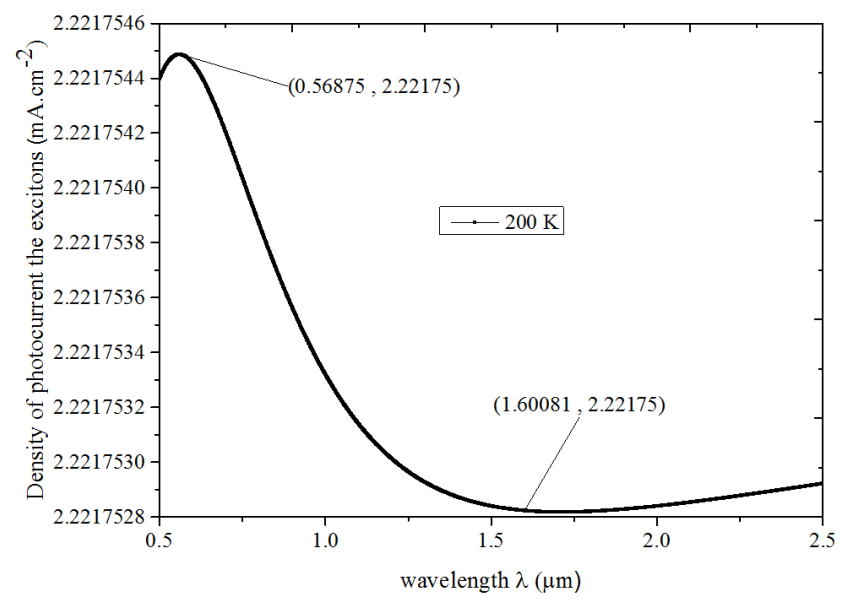

Figure 13. Exciton photocurrent density as a function of wavelength $(\lambda)$ at $T=200 K$

Contrary to what is often explained in the literature, crystalline silicon has a range of wavelengths encompassing its gap energy $E_{g}=\frac{h . c}{\lambda_{g}} .\left(0,6 \mu m \prec \lambda_{g} \prec 1,6 \mu m\right)$, in which it absorbs photons. But photons of energies greater than the energy of the gap $\left(\lambda \prec \lambda_{g}\right)$ generate more load carriers because they have enough energy to transfer electrons from the valence band to the conduction band. Whereas photons of energies less than the energy of the gap $\left(\lambda \succ \lambda_{g}\right)$ do not have enough energy, but since they have an energy close to the energy of the gap, there are excited levels between the bands of conduction and valence. They are able to attract electrons that have less energy than the gap. In addition to the phenomena that make it possible for the carriers to pass from one level of energy to the other, the charged electrons interact with each other during their transitions, for example they repel each other, exchange energies among themselves. There are forces like the electric field that deflects them from their trajectories.

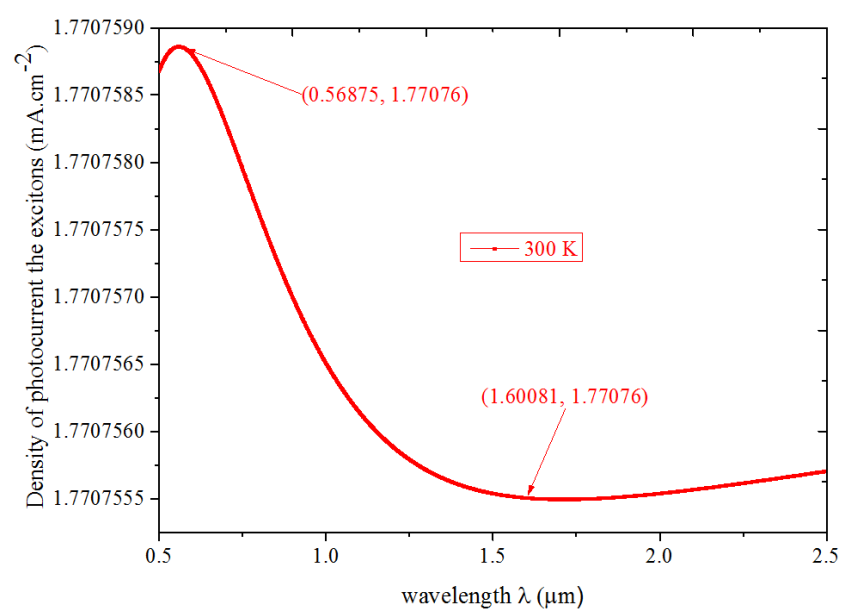

Figure 14. Exciton photocurrent density as a function of wavelength $(\lambda)$ at $T=300 \mathrm{~K}$

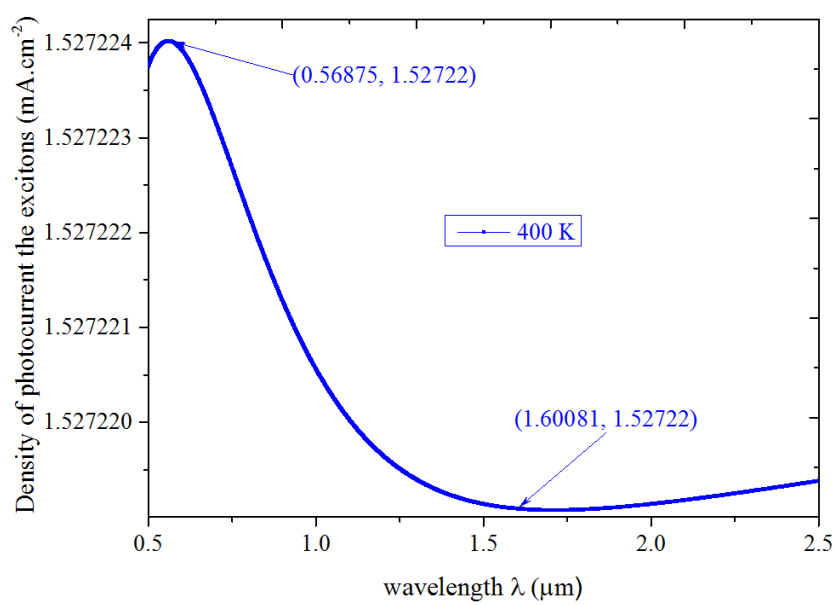

Figure 15. Exciton photocurrent density as a function of wavelength $(\lambda)$ at $T=400 K$

For the movement of the load carriers to be orderly, it is necessary that electrons that are in stable positions or that have sufficient energy to reach permissible or intermediate energy levels (excitonic levels) have no influence. In addition, a thermal excitation creates disorder in crystalline silicon by bringing stable electrons out of the valence band of their orbits: that is to say, they are in interstitial positions and this only occurs at very high temperatures. This disorder creates recombinant sites that attract excitons and trap them. On the other hand, an increase in temperature also results in a decrease in the absorption gap, facilitating the orderly movement of free electrons. 


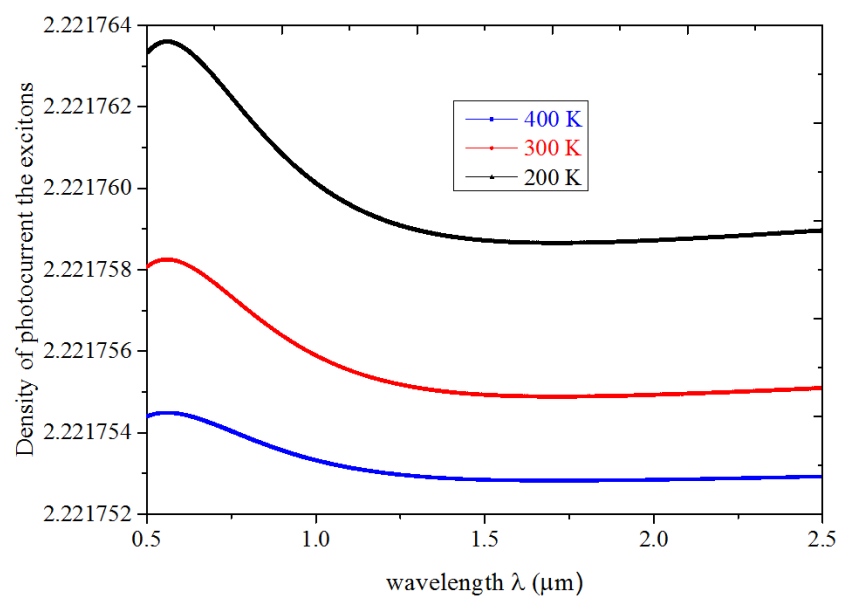

Figure 16. Exciton photocurrent density as a function of wavelength $(\lambda)$ for different temperature values.

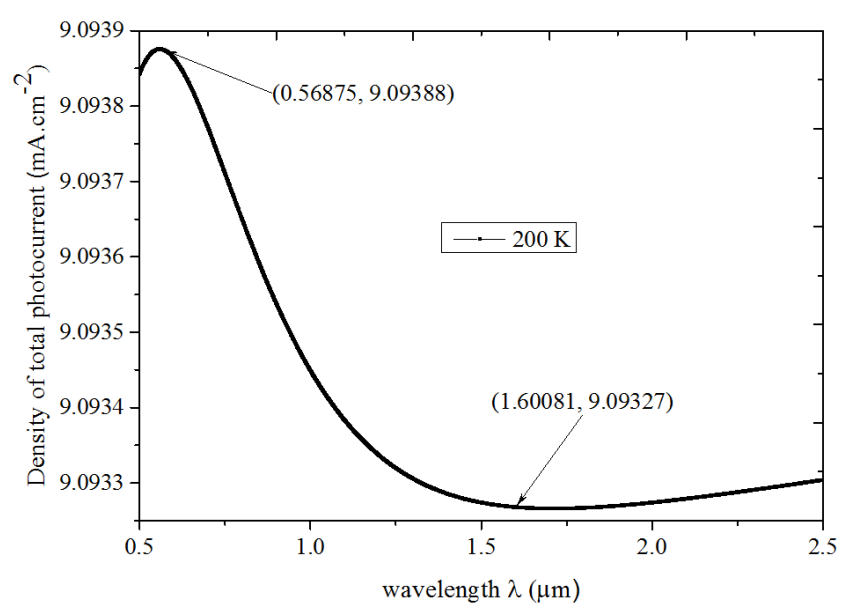

Figure 17. The total photocurrent density as a function of wavelength $(\lambda)$ at $T=200 \mathrm{~K}$.

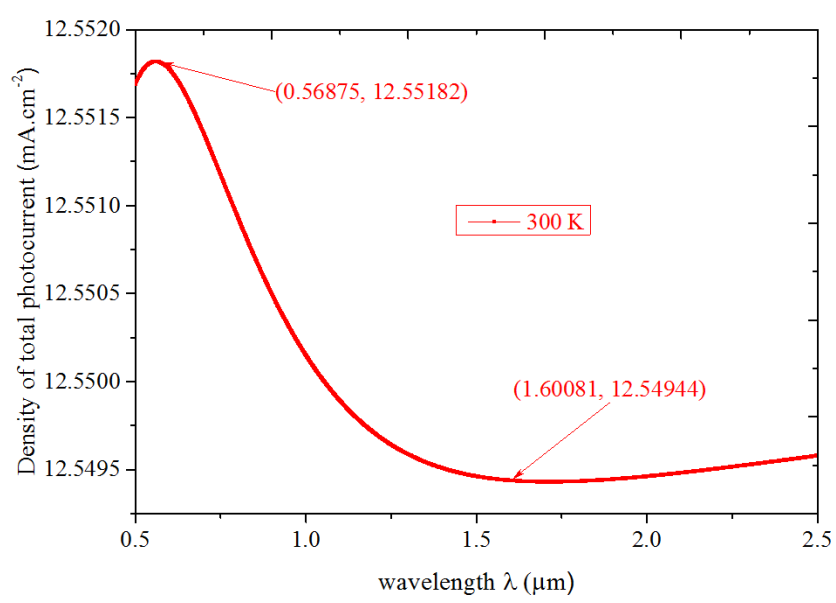

Figure 18. The total photocurrent density as a function of wavelength $(\lambda)$ at $T=300 \mathrm{~K}$.

On the other hand, at temperature values ranging from $100 \mathrm{~K}$ à $500 \mathrm{~K}$, there is an increase in the photocurrent density of the electrons and a decrease in that of the excitons (Figures 12 and 16). The total photocurrent density (electrons and excitons) varies in the same way as that of electrons, but with a difference of 2,22 $\mathrm{mA}_{\mathrm{cm}} \mathrm{cm}^{-2}$ (Figure 20).

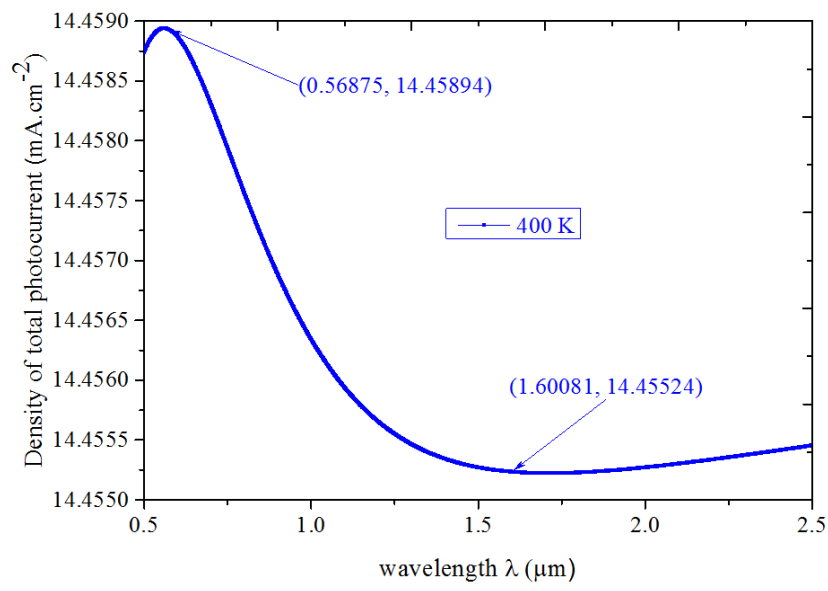

Figure 19. The total photocurrent density as a function of wavelength $(\lambda)$ at $T=400 \mathrm{~K}$.

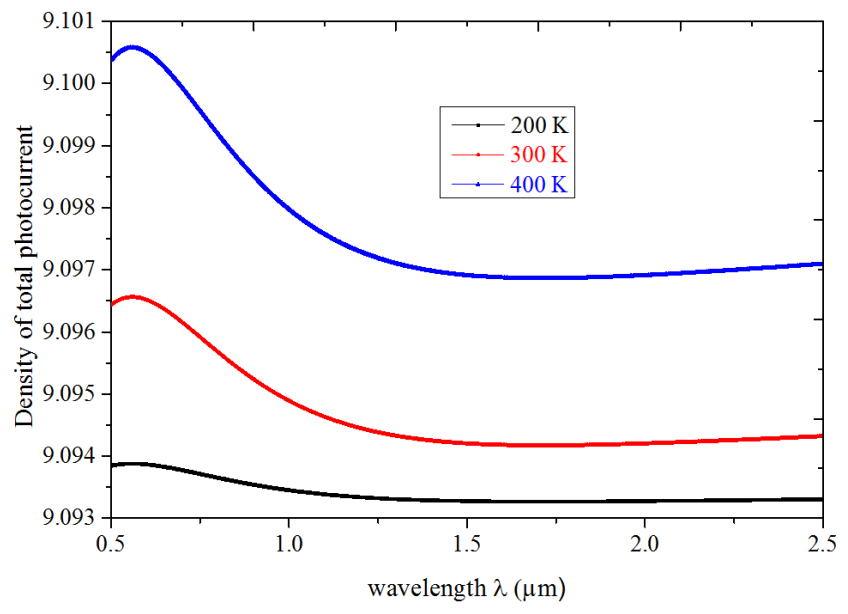

Figure 20. The total photocurrent density as a function of wavelength $(\lambda)$ for different temperature values.

Thus, we can say that as long as we do not reach temperatures that exceed the critical value for the material used, the temperature can be considered favourable to the photocurrent density.

But here too, we cannot say with certainty that the temperature is favourable to the efficiency of our photovoltaic cell.

To do this, we will use the calculation of internal quantum efficiency to measure the efficiency of the photovoltaic cell illuminated by a polychromatic light with wavelengths between $0,6 \mu \mathrm{m}$ et $2,5 \mu \mathrm{m}$.

In this section, we were able to define a very precise wavelength range between $0,6 \mu \mathrm{m}$ et $1,6 \mu \mathrm{m}$, in which we have variations in photocurrent density.

\subsection{The Internal Quantum Efficiency of the Photovoltaic Cell}

In the case of quantum solar cell yields, transparent and conductive oxides (TCO), considered as window layers, are highlighted. Indium oxide (ITO) is used in our solar cell as transparent electrodes. It has a high optical transmission due to its high gap, good electrical conductivity which allows it to obtain the least transport losses of photogenerated charges and low reflection. This allows it to efficiently transport the 
photons to the active layer. The photocurrent density study allowed us to define a wavelength range between $0,6 \mu \mathrm{m}$ et $1,6 \mu \mathrm{m}$ in which the c-Si (p) is more active. Photons passing through the active layer by photogeneration undergo recombination and dissociation phenomena that are at the origin of losses in the base.

In Figures 21-23 we reproduce the internal quantum yield of electrons, that of excitons and the total internal quantum yield as a function of the wavelength for different temperature values.

The curves in Figures 21-23 show, for wavelengths between $0,6 \mu \mathrm{m}$ and $2,5 \mu \mathrm{m}$, an increase in efficiency up to a value of the wavelength $\lambda=1,5 \mu \mathrm{m}$ then a progressive decrease.

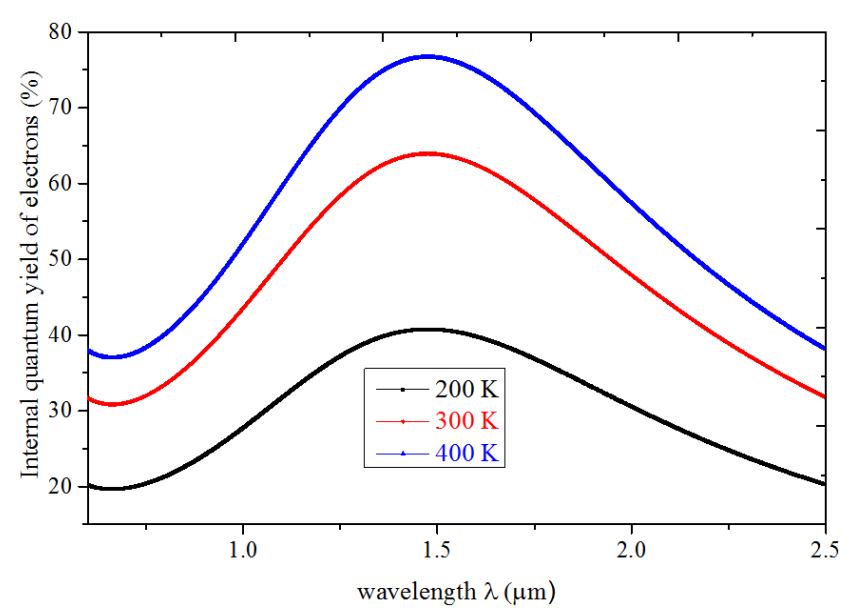

Figure 21. The internal quantum yield of electrons as a function of wavelength for different temperature values.

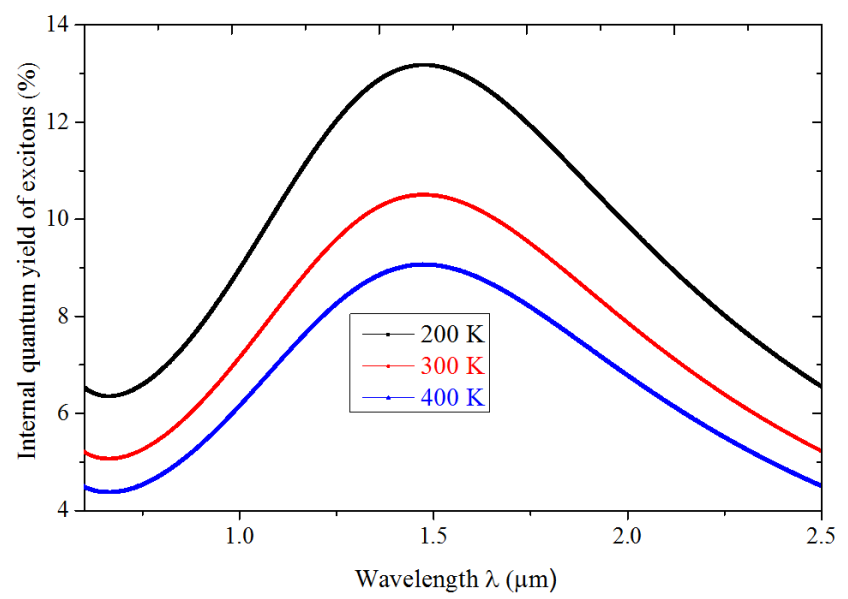

Figure 22. The internal quantum yield of excitons as a function of wavelength for different temperature values.

In other words, an increase in temperature leads to an increase in the internal quantum yield of electrons and a decrease in the yield of excitons (Figures 21 and 22).

Silicon absorbs photons with wavelengths between $0,6 \mu \mathrm{m}$ et $1,6 \mu \mathrm{m}$. Beyond this wavelength range, the photocurrent density, with a minimum value, remains virtually invariant. It absorbs and transmits photons of wavelengths close to the energy of the absorption gap $\left(0,6 \mu m \prec \lambda_{g} \prec 1,6 \mu m\right)$ by photogeneration. These photons generating carriers can suffer losses by recombination. These recombinations are: volumic in the photovoltaic cell and surfacing at the interface between a-Si: $\mathrm{H} \mathrm{(n)/c-Si} \mathrm{(p)} \mathrm{and} \mathrm{the}$ front and rear sides.

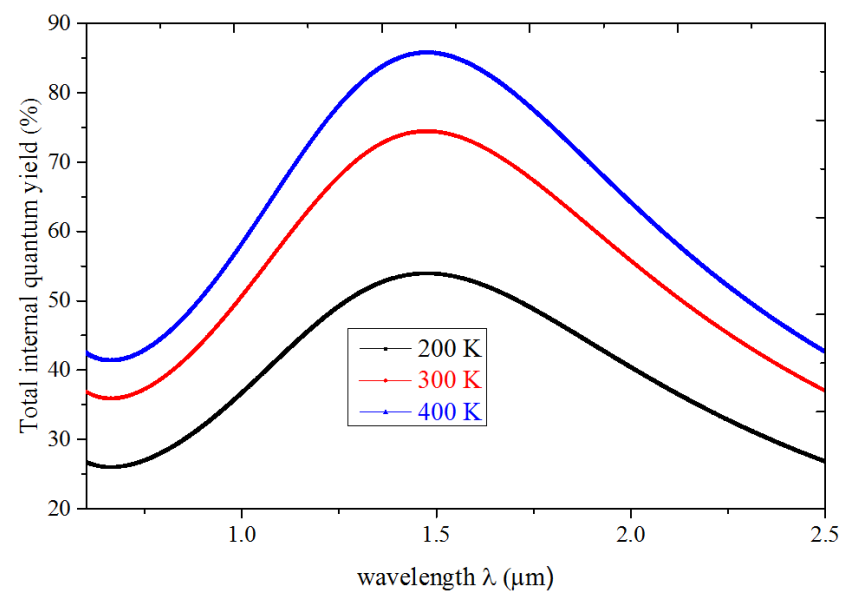

Figure 23. Total internal quantum yield as a function of wavelength for different temperature values.

At a wavelength $\lambda=1,5 \mu \mathrm{m}$ value that corresponds to the plasma wavelength, most photons have low energy that does not allow them to pass through the transparent indium oxide (ITO) window layer.

Two very interesting effects of temperature lead to antagonistic results on the internal quantum performance of electrons and excitons. The increase in temperature leads to a decrease in the energy of the gap which is favourable to the internal quantum efficiency of electrons while a thermal excitation is unfavourable to the internal quantum efficiency of excitons.

The curves in Figure 23 show an increase in the total internal quantum efficiency of the photovoltaic cell of $10 \%$ for the different temperature values. This increase is due to the intake of excitons.

\section{Conclusion}

Our study shows that excitons are more stable and diffusible than electrons at very low temperatures. This is confirmed by researchers in this field [13-15]. In addition, a disorderly increase in carrier density was observed. Thus, the results obtained on the densities of, photocurrent allowed us to define a wavelength range between $600 \mathrm{~nm}$ and $1600 \mathrm{~nm}$ in which the $\mathrm{c}-\mathrm{Si}(\mathrm{p})$ is more active to generate the load carriers. Finally, we obtained quantum yields of around $80 \%$ with a $10 \%$ participation of excitons for the different temperature values.

\section{References}

[1] M. Faye, M. Niane, S. Ndiaye, O. Ngom, C. Mbow, B. Ba; Numerical Modeling of Effects of Excitons on Photoelectric Properties of Cells; Journal of Scientific and Engineering Research volume 6 (6) (2019) 138-146. 
[2] M. Burgelman and B. Minnaert; Including excitons in semiconductor solar cell modelling; Thin Solid Films 511-512, 214-218 (2006).

[3] S. Zh. Karazhanov; Temperature and doping level dependence solar cell performance Including excitons. Solar Energy Materials \& Solar Cells 63 (2000) 149-163.

[4] M. Faye, C. Mbow, B. Ba; Internal Electric Field In The Space Charge Layer Of A Solar Cell Based On Silicon In The Presence Of Excitons; International journal of scientific \& technology research volume 4 (2015) 66-69.

[5] M. Faye, M. Niane, S. Ndiaye, C. Mbow, B. Ba; Effects of Variability of The Average Temperature on The Distribution of Electrons and of Excitons in A Semiconductor; Journal of Materials Science \& Surface Engineering; volume 4 (7) (2016) 467-471.

[6] A. Armin, D. M. Stoltzfus, J. E. Donaghey, A. J. Clulow, R. C. R. Nagiri, P. L. Burn, I. R. Gentle and P. Meredith; Engineering dielectric constants in organic semiconductors; The Royal Society of Chemistry (2017).

[7] N. Hiroshiba, K. Morimoto, R. Hayakawa, Y. Wakayama, T. Mori, and K. Matsuishi; Exciton dynamics at the heteromolecular interface between $\mathrm{N}$, N'-dioctyl-3,4,9,10-perylenedicarboxilimide and quaterrylene studied using time-resolved photoluminescence; AIP ADVANCES 4, 067112 (2014).

[8] Oleksandr V. Mikhnenko, Paul W. M. Blom, Thuc-Quyen Nguyen; Exciton Diffusion in Organic Semiconductors; Energy \& Environmental Science 1-64 (2015).
[9] M. K. Riede, T. Mueller, B. Maennig, K. Leo, K. O. Sylvester-Hvid, B. Zimmermann, M. Niggemann and A. Gombert; Comment on "Roles of donor and acceptor nanodomains in $6 \%$ efficient thermally annealed polymer photovoltaics"; Appl. Phys. Lett. 90, 163511 (2007).

[10] S. Blumstengel, S. Sadofev, C. Xu, J. Puls, and F. Henneberger; Converting Wannier into Frenkel Excitons in an Inorganic/Organic Hybrid Semiconductor Nanostructure; Physical review letters; PRL 97, 237401 (2006).

[11] Hangleiter, R. Hacker; Enhancement of band-to-band Auger recombination by electron-hole correlations; Phys. Rev. Lett. 65, 215 (1990).

[12] Patankar, S. V.; Numerical Heat Transfer and Fluid Flow. Hemi- sphere Publishing Corporation, Taylor and Francis Group, New York 1980.

[13] C. Klingshirn, H. Kalt, M. Umlauff, W. Petri, F. A. Majumder, S. V. Bogdanov, W. Langbein, M. Grün, M. Hetterich, K. P. Geyzers, M. Heuken, A. Naumov, H. Stanzl, W. Gebhardt, "Stimulated emission of II-VI epitaxial layers", J. Cryst. Growth 138, 786, (1994) p. 786-790.

[14] D. C. Reynolds, C. W. Litton, T. C. Collins, "Zeeman effects in the edge emission and absorption of ZnO", Phys. Rev. 140, A1726, (1965) 1726-1734.

[15] J. M. Hvam, "Exciton-exciton interaction and laser emission in high-purity ZnO”, Solid State Commun. 12, 95-97, (1973). 